CHERCHER, REPÉRER, AVANCER

\title{
LA CIRCULATION CROISÉE
}

Modèles, labels et bonnes pratiques dans les rapports centre-périphérie

Vincent Béal, Renaud Epstein, Gilles Pinson

Presses de Sciences Po (P.F.N.S.P.) | « Gouvernement et action publique »

2015/3 N 3 | pages 103 à 127

ISSN 2260-0965

ISBN 9782724634044

Article disponible en ligne à l'adresse :

http://www.cairn.info/revue-gouvernement-et-action-publique-2015-3-page-103.htm

\section{Pour citer cet article :}

Vincent Béal et al., « La circulation croisée. Modèles, labels et bonnes pratiques dans les rapports centre-périphérie », Gouvernement et action publique 2015/3 (N 3), p. 103-127.

DOI 10.3917/gap.153.0103

Distribution électronique Cairn.info pour Presses de Sciences Po (P.F.N.S.P.).

(C) Presses de Sciences Po (P.F.N.S.P.). Tous droits réservés pour tous pays.

La reproduction ou représentation de cet article, notamment par photocopie, n'est autorisée que dans les limites des conditions générales d'utilisation du site ou, le cas échéant, des conditions générales de la licence souscrite par votre établissement. Toute autre reproduction ou représentation, en tout ou partie, sous quelque forme et de quelque manière que ce soit, est interdite sauf accord préalable et écrit de l'éditeur, en dehors des cas prévus par la législation en vigueur en France. Il est précisé que son stockage dans une base de données est également interdit. 


\title{
LA CIRCULATION CROISÉE
}

\section{Modèles, labels et bonnes pratiques dans les rapports centre-périphérie}

\author{
Vincent Béal, Renaud Epstein et Gilles Pinson
}

Résumé : Cet article s'intéresse aux circulations de modèles et autres «bonnes pratiques " de politique urbaine et à leur rôle dans la reconfiguration des rapports entre l'État et les villes. II montre que l'intensification des circulations de pratiques, de savoirs et d'expériences dans les politiques urbaines ne peut être réduite à des dynamiques d'horizontalisation et de transnationalisation de l'action publique urbaine. Elle doit également être comprise au regard de la restructuration de l'État et de son intervention territoriale. En s'appuyant sur une analyse du Programme nationale de rénovation urbaine et du plan Ville durable, l'article souligne l'émergence d'un nouveau modèle de relations centre-périphérie fondé sur le repérage par l'État d'expériences locales « innovantes » ou « exemplaires », puis leur validation et promotion par la labellisation, l'inscription dans des répertoires de "bonnes pratiques " et autres formes de mise en visibilité. Ce modèle de circulation croisée permet à l'État de retrouver des capacités de mobilisation et d'orientation à distance des politiques menées localement, sans pour autant remettre en cause le mouvement de montée en puissance des villes.

MOTS-CLÉS : CIRCULATIONS - DÉVELOPPEMENT DURABLE - ÉTAT - GOUVERNANCE URBAINE MODĖLES - POLITIQUES URBAINES - RAPPORTS CENTRE-PÉRIPHÉRIE - RÉNOVATIONS URBAINES TRANSFERTS DE POLITIQUE PUBLIQUE

\section{CROSSED CIRCULATION: MODELS, AWARDS AND BEST PRACTICES IN CEN- TRAL-LOCAL RELATIONSHIPS}

Abstract: This article focuses on the circulation of urban policy models and "best practices" and its influence on the reshaping of relationships between States and local governments. It suggests that the increasing mobility of practices, knowledge and initiatives in urban policies can not only be explained by the horizontal and transnational dynamics of urban policy-making. It relies also on the restructuring of the State the ways in which its policies are formulated and implemented at the local levels. By considering the National Programme for Urban Renewal and the Sustainable City Plan, the article highlights the emergence of a new model of central-local relationships based on the State' tracking of local "innovative" or "exemplary" initiatives, and on their certification and diffusion. This model of crossed circulation allows the State to strengthen its capacity to mobilize and steer at a distance urban policies, without actually challenging the rise in power of cities.

KEYWORDS: CENTRAL-LOCAL RELATIONSHIPS - GOVERNANCE - POLICY TRANSFER - POLICY MOBILITIES - SUSTAINABLE DEVELOPMENT - URBAN POLICIES - URBAN RENEWAL 
Depuis quelques années, l'action publique urbaine est marquée par une explosion des phénomènes de circulation de savoirs, d'expertises, d'expériences, d'instruments, de pratiques et de modèles, procédant d'échanges bilatéraux entre villes et/ou de la médiation d'acteurs ou d'organisations tiers ${ }^{1}$. Dans un contexte de globalisation, d'intensification de la compétition entre les territoires et de restructuration des États, les acteurs locaux se tournent de façon croissante vers des expériences exogènes - souvent identifiées comme des «modèles " ou des "bonnes pratiques " - pour construire les stratégies et politiques urbaines. Cette évolution s'est accompagnée de la constitution, aux échelles nationale et internationale, d'un véritable secteur de la consultance propre aux questions urbaines et de la multiplication des réseaux des villes, voyages d'études, salons et congrès ou encore guides de «bonnes pratiques » qui fonctionnent comme des accélérateurs de la diffusion et de la circulation des politiques urbaines considérées comme innovantes ou exemplaires. Elle est le signe d'une transformation de l'action publique urbaine, certes marquée par une montée des dynamiques transnationales, mais aussi, voire surtout, par une recomposition des rapports entre l'État et les villes qui sont structurés de manière croissante par des processus de sélection, de certification et de diffusion de modèles de politique urbaine.

Alors que l'étude des transferts et des circulations aux échelles nationales et surtout internationales constitue aujourd'hui un champ bien balisé de la science politique (Dolowitz, Marsh, 2000 ; Evans, 2004 ; Delpeuch, 2008), celle des échanges entre villes ou plus généralement entre espaces politiques locaux est beaucoup moins développée. Dans ce domaine, il faut souvent se tourner vers les travaux de géographie ou d'urbanisme pour trouver des analyses de la circulation des politiques et pratiques locales (Devisme et al., 2007 ; Soderstrom, Paquot, 2013 ; Carriou, Ratouis, 2014). La géographie radicale anglosaxonne s'est montrée elle aussi particulièrement attentive à cette évolution. Tout un chantier de recherche s'est développé autour de la notion de "policy mobilities " (McCann, Ward, 2011 ; Peck, 2011 ; Robinson, 2011 ; Peyroux et al., 2012 ; Peck, Theodore, 2015). Pour ces travaux, les circulations sont le révélateur d'une transformation profonde des villes et du contexte dans lequel elles évoluent. En l'espace de quelques années, les villes sont devenues des points d'ancrages essentiels des dynamiques de la globalisation néo-libérale (Brenner, 2004). Traversées par des flux d'informations et d'influences, elles seraient désormais le terrain de jeu de compagnies transnationales, de consultants nomades et d'institutions supranationales capables d'imposer leurs normes et de prescrire le contenu des politiques urbaines. Ainsi, ces travaux donnent à voir l'émergence d'une action publique façonnée par des processus hors-sol d'import/export. Les géographes mobilisent souvent la notion

1. En utilisant la notion de circulation pour qualifier l'ensemble de ces échanges, plutôt que celle de transfert, nous nous situons dans le sillage de travaux existants de politistes (Oliver, Lodge, 2003 ; Hassenteufel, 2005 ; Kaluszynski, Payre, 2013) et de géographes (McCann, Ward, 2001, 2012 ; Peck, 2011; Robinson, 2011). Comme eux, nous considérons que la notion de transfert charrie une vision par trop mécanique et désincarnée des mécanismes de diffusion et d'influence. La notion de circulations, comme celle de policy mobilities utilisée par les géographes, s'inscrit dans des cadres analytiques plus sensibles aux acteurs, aux communautés politiques, administratives, épistémiques et professionnelles dans lesquels ils s'inscrivent, à leurs ressources, à leurs positions et au travail politique qu'ils doivent consentir pour diffuser, acclimater et adapter des " solutions " venues d'ailleurs. La notion de circulation nous semble aussi plus sensible à ce que la mise en mouvement fait aux idées, modèles et instruments, bref aux phénomènes d'altération et d'hybridation qui peuvent s'opérer à la faveur d'un désencastrement du territoire d'origine et d'un réencastrement dans le territoire d'importation. 
deleuzienne d'assemblage pour décrire cette situation nouvelle où l'élaboration des politiques urbaines mêlerait des logiques de déterritorialisation, autour de la mobilisation d'expériences exogènes, et de reterritorialisation au travers de luttes politiques locales pour la réinterprétation de ces éléments « importés » (McCann, Ward, 2011 ; McFarlane, 2011).

Si ces travaux de géographie et d'urbanisme ont le mérite de souligner l'influence des circulations horizontales dans la production des politiques urbaines et le rôle des villes dans la construction de réseaux globalisés d'échanges, ce que font beaucoup plus rarement les travaux de science politique, ils ont le défaut de laisser de côté le rôle de l'État dans ces circulations. Tout à leur entreprise de revalorisation du rôle politique des villes, ils postulent implicitement le basculement des villes dans une ère d'horizontalisation généralisée qui parachèverait un mouvement de dénationalisation et de désétatisation des politiques urbaines (Benson, Jordan, 2012 ; Béal, 2014). Les dynamiques transnationales court-circuiteraient ainsi les espaces politiques nationaux dans lesquels les "vieux » États territoriaux, incapables de s'adapter aux nouvelles logiques de production des politiques urbaines, auraient perdu une grande part de leur influence sur les villes et les territoires (McCann, Ward, 2012). C'est précisément ce point que cet article entend discuter. II s'agit de montrer que l'intensification des circulations dans les politiques urbaines doit être analysée non seulement de façon horizontale mais aussi verticale, en considérant le rôle de l'État (et de l'échelle nationale) dans la mise en circulation et la diffusion de modèles, de pratiques, d'objectifs, rôle que les géographes et urbanistes ont tendance à négliger. L'État reste un acteur nodal des politiques urbaines et ses interventions dans ce domaine se sont en partie restructurées autour de la stimulation et du cadrage des circulations comme levier d'orientation des politiques menées dans les villes.

La réintroduction de l'État dans l'analyse des circulations de politiques publiques entre villes n'a pas pour seul mérite d'éclairer la nature de ces échanges. Elle a aussi l'intérêt de rouvrir le dossier des rapports centre-périphérie en France en réarticulant deux ensembles de travaux qui ont eu tendance ces dernières années à s'ignorer mutuellement, au détriment d'une juste appréhension de l'évolution des rapports entre l'État et les villes. Le premier ensemble est constitué par les études portant sur la recomposition de l'État. Ces travaux donnent à voir les ruses, le plus souvent d'inspiration néomanagériale, de l'État pour se repositionner dans les politiques territoriales et rogner l'autonomie chèrement acquise par les gouvernements locaux et régionaux (Epstein, 2013a; Bezes, Le Lidec, 2011a, 2011b). Les seconds, regroupés dans le chantier de recherche de la gouvernance urbaine, décrivent les processus de renforcement de la capacité d'action des villes et des métropoles et la manière dont elles s'affranchissent progressivement non seulement de la tutelle du centre mais aussi des ressources, notamment d'expertise, dont il était jusque récemment le pourvoyeur essentiel (Le Galès, 2003, p. 11 ; Pinson, 2009). Notre étude nous conduit à conclure que ces deux phénomènes ne sont en réalité pas contradictoires mais plutôt intimement imbriqués et qu'ils contribuent ensemble à redéfinir assez nettement le modèle français de rapport centre-périphérie. Certes, la capacité de l'État à intervenir dans les territoires s'est nettement amenuisée (Dupuy, Pollard, 2014), mais son influence procède désormais de formes d'intervention moins directes, qui passent, entre autres, par l'identification, la sélection, la modélisation, la valorisation et la diffusion des expériences « innovantes » ou " exemplaires » des gouvernements urbains. Cette recomposition de l'intervention territoriale de 
l'État - que nous qualifions ici de « circulation croisée » en clin d'œil au modèle de la « régulation croisée " qui dominait les rapports centre-périphérie jusqu'aux années 1980 (Crozier, Thoenig, 1975) - s'appuie sur des instruments spécifiques qui empruntent à la figure de l'État régulateur : appels à projet compétitifs, benchmarking, indicateurs de performance, labels, mise en réseau, etc. (Moran, 2002 ; Hassenteufel, 2008 ; Lascoumes, Le Galès, 2004 ; Le Galès, 2013). Ces instruments ont permis à l'État de rétablir une part d'influence sur les villes en stimulant la compétition interurbaine et en prescrivant des contenus de politiques urbaines (agendas, normes d'organisation, priorités d'action, etc.), sans pour autant entraver la montée en puissance des villes, de leur capacité d'expertise et d'action mais aussi de leur faculté à établir des relations horizontales avec d'autres villes, y compris au-delà des frontières nationales.

La démonstration empirique s'appuie sur l'analyse des politiques de rénovation urbaine et de développement urbain durable. Plus précisément, cette analyse porte sur deux programmes $^{2}$ : le Programme national de rénovation urbaine (PNRU), initié en 2003 pour organiser la démolition/reconstruction des quartiers de grands ensembles ; le plan Ville durable, lancé en 2008 et complété par le fonds Ville de demain, pour faire émerger des formes de production urbaine plus soucieuses de l'environnement. Le choix de ces deux programmes s'appuie sur quatre critères. II s'agit tout d'abord de programmes relativement récents portés par des institutions emblématiques des réformes récentes de l'administration française et des rapports centre-périphérie : I'Agence nationale de la rénovation urbaine (ANRU) pour le PNRU et le "super ministère " de l'Environnement, la Caisse des dépôts et consignations $(\mathrm{CDC})$ et le Commissariat général à l'investissement (CGI) pour le plan Ville Durable. II s'agit ensuite de programmes qui se démarquent des formes traditionnelles de gouvernement (de type " command and control "), pour privilégier des instruments incitatifs et des mécanismes d'autocontrôle supposés, en bonne logique néomanagériale, accroître la performance de l'action publique. Par ailleurs, il s'agit de deux programmes qui ont bénéficié d'une forte visibilité et qui ont été construits autour d'une rhétorique volontariste, accordant une large place aux enjeux d'innovation et d'exemplarité. Enfin, les deux programmes ont des équivalents dans de nombreux pays européens (voire au-delà). Ils constituent donc de bons terrains pour établir si les circulations en la matière s'effectuent principalement dans un cadre transnational ou davantage national.

Dans la première partie de cet article, à dominante théorique et historique, nous décrivons les deux dynamiques, a priori contradictoires mais en réalité articulées, qui ont travaillé à la fois les circulations des savoirs et des modèles en matière de politique urbaine et la structure des rapports entre l'État et les villes depuis une quarantaine d'années : la montée en puissance des circulations interurbaines d'une part, et le repositionnement de l'État comme orchestrateur de ces circulations d'autre part. Dans la seconde partie, plus empirique, nous présentons les deux programmes susnommés. lls nous permettent de montrer concrètement de quelle manière l'État a délégué aux villes la responsabilité de concevoir et mettre en œuvre les projets du PNRU et du plan Ville durable, mais aussi comment ces

2. Ces deux programmes ont été étudiés dans le cadre de différents projets de recherche engagés au cours de ces dernières années, et plus particulièrement à l'occasion de deux recherches doctorales, l'une en sociologie (Epstein, 2008) et l'autre en science politique (Béal, 2011). 
projets s'alimentent d'une intense activité d'échanges entre villes. En même temps, ces circulations s'opèrent essentiellement dans un cadre national et sont en grande partie organisées par l'État. II s'agit donc moins de circulations horizontales stricto sensu que de mécanismes dont les termes anglais d'uploading et de downloading permettent de rendre raison. Ces mécanismes, qui prennent la forme du repérage par l'État de bonnes pratiques puis de leur validation et promotion par la labellisation, l'inscription dans des répertoires de bonnes pratiques et autres formes de mise en avant de "villes modèles ", permettent au centre de retrouver des capacités de mobilisation et d'orientation à distance des politiques menées localement.

\section{La boucle des transferts de politique urbaine}

Dans cette première partie, nous voudrions, à partir du cas français, contribuer au dépassement de deux biais de la littérature sur les circulations des modèles de politique publique. Celui consistant, du côté de la science politique, à de très rares exceptions près (Wolman, Page, 2002), à concentrer l'attention sur les circulations de modèles et de pratiques entre espaces nationaux ou sur les transferts imposés par des organisations supranationales à des États. Celui consistant, du côté de la géographie, à passer l'échelon stato-national par pertes et profits au nom de la réhabilitation des transferts interurbains et du dépassement du « nationalisme méthodologique ». C'est seulement à ce prix qu'il est possible de mettre l'analyse des circulations au service d'une lecture lucide et mesurée des transformations des rapports entre les villes et les échelons supérieurs de gouvernement. Dans un premier temps, nous nous pencherons sur le développement des circulations horizontales dans lesquelles les villes françaises sont de plus en plus impliquées. Dans un second temps, nous nous intéresserons à l'État pour voir de quelles manières il a su, en même temps qu'il perdait de sa centralité dans les politiques urbaines, reconstruire une place nodale dans les mécanismes de circulation et réintroduire ainsi une dose de verticalité dans l'analyse de ces circulations.

\section{Vers une horizontalisation des transferts ?}

En France, les trente dernières années ont été marquées par une montée en puissance des circulations horizontales impliquant les gouvernements urbains et métropolitains. Parfois spontanées, ces circulations s'opèrent le plus souvent au travers de la médiation d'acteurs ou d'organisations tiers: des professionnels, des consultants, des réseaux de coopération nationaux ou transnationaux, des associations professionnelles mais aussi des partis politiques qui peuvent offrir des canaux de circulations professionnelles pour les hauts fonctionnaires et les élus urbains qui deviennent autant de vecteurs de mise en circulation de modèles d'action publique. Dans ces circulations horizontales, le degré de contrainte est relativement faible. Rappelons que Dolowitz et Marsh (2000) ont proposé une typologie de transferts fondée sur la mesure du degré de contrainte sous-tendant les circulations. Cette typologie distingue 1) les transferts directs contraints impliquant généralement une organisation supranationale imposant des normes ou des modèles à des pays ou des gouvernements infranationaux ; 2) les transferts indirects contraints dans lesquels c'est plutôt la perception d'une 
contrainte qui amène des gouvernements occupant une position dominée à adopter les options retenues par des tiers dominants ; 3) les transferts volontaires reposant sur la liberté des « importateurs » de choisir leurs sources d'inspiration. Les circulations horizontales entre villes relèvent davantage des deux derniers types. L'acclimatation de nouvelles manières de formuler les problèmes, de concevoir et de mettre en œuvre des politiques urbaines opère largement au travers de la socialisation des acteurs dans le cadre d'associations, de forums, de congrès, de voyages d'étude ou au travers de l'influence exercée par les modèles véhiculés par les consultants, la presse spécialisée ou grand public.

Ces circulations sont-elles chose tout à fait nouvelle s'agissant des gouvernements urbains ? Certes non. Les travaux d'histoire et de socio-histoire portant sur les villes ont montré comment certaines périodes historiques ont été propices au développement de ce type de circulations. Saunier parle de «toile municipale " pour qualifier "un espace social qui traverse les limites nationales, régionales ou linguistiques et dont l'étendue est définie par l'extension des flux d'information, d'idées, de procédés, de services, de personnes centrés sur les pratiques, les formes, les méthodes, les principes et les valeurs du gouvernement municipal des villes » et qui se déploie avec force à la charnière des $19^{\circ}$ et $20^{\circ}$ siècles (Saunier, 2006, p. 43). Pour lui, l'observation des circulations denses qui associent dès cette époque les villes conduit à nuancer l'idée d'une nouveauté des réseaux et des échanges urbains qui se déploient à partir des années 1980. "Globales, écrit Saunier, les villes le sont alors par les projets, les relations, les pratiques que révèle l'observation de la toile municipale» (2006, p. 44).

Toutefois, ces travaux socio-historiques reconnaissent que ces circulations horizontales ont connu des périodes d'éclipses correspondant aux moments où la souveraineté municipale est rognée par les États territoriaux. C'est dans une période écliptique de ce type que les gouvernements urbains français rentrent à la fin de la Troisième République pour n'en sortir que dans les années 1970. La censure du Conseil d'État et le progressif retour en force du ministère de l'Intérieur et du corps préfectoral à la fin de la Troisième République, puis les logiques centralisatrices impulsées, en matière d'urbanisme notamment, par le gouvernement de Vichy (Gaudin, 1985 ; Claude, Saunier, 1999) commencent à limiter les marges de manœuvre en matière de coopération municipale transnationale mais aussi à priver les gouvernements urbains de compétences (urbanisme, transports, assainissement) pour lesquelles s'exprimait un besoin d'échanges. Cette tendance s'accentue au cours des Trente Glorieuses, avec la montée en puissance de l'État planificateur, aménageur et urbaniste qui contribue à geler les circulations interurbaines domestiques et transnationales. Les travaux du Centre de sociologie des organisations ont mis au jour les ressorts de cet assèchement des circulations horizontales (Crozier, Thoenig, 1975; Grémion, 1976 ; Thoenig, 1973 ; Borraz, 1998). D'une part, la prise en charge directe de l'essentiel des politiques urbaines par l'État, ses préfets, ses services déconcentrés et son réseau scientifique et technique tend à exclure les élus de la conception de ces politiques et à les reléguer dans un rôle de courtier en influence (Lorrain, 1991). Ce rôle les dispose davantage à chercher à capter des ressources ou à obtenir des arrangements auprès des services du préfet et des ministères qu'à fréquenter des forums d'échange d'expériences ou d'expertise. Cela n'est pas un hasard si, à cette époque, l'action internationale des villes se réorganise autour des jumelages. Privés des leviers d'action sur l'espace urbain, les édiles investissent des enjeux plus 
lointains comme la paix et la construction européenne (Vion, 2003). D'autre part, la concentration de l'expertise technique et des ressources financières au sein des services de l'État, associée aux contraintes fortes qui pèsent sur l'embauche par les gouvernements urbains de personnels qualifiés, tend à affaiblir l'expertise municipale et à faire des techniciens des villes les supplétifs des services déconcentrés de l'État. Dans ces conditions, il est difficile de parler de circulations des savoirs, des modèles, des pratiques et de l'expertise puisque l'État, au travers de ses administrations centrales et déconcentrées, est à la fois en charge du design des modèles et de leur mise en œuvre dans les territoires. Si circulation il y a, elle opère au sein des administrations étatiques et contribue à l'uniformisation des politiques urbaines.

II faut attendre les années 1970 et les deux décennies suivantes pour assister à une relance des circulations horizontales autour des enjeux de politique urbaine. Ces circulations ont d'abord pour terreau la contestation des politiques urbaines de l'État et de l'expertise des ingénieurs des Ponts et Chaussées (IPC) qui, depuis la création du ministère de l'Équipement en 1966, ont la haute main sur ce secteur (Thoenig, 1973). Paradoxalement, c'est souvent dans des réseaux mis en place par l'État lui-même qu'une expertise alternative va se constituer, circuler entre villes pour finalement servir de contre-projet à la politique de l'État en matière d'aménagement urbain. Offner (1988) relate par exemple la constitution d'une expertise en transports urbains dans les agences d'urbanisme, les centres d'études techniques de l'équipement (CETE) et les entreprises locales de transports urbains. Cette expertise se constitue bientôt en alternative aux visions routières des IPC et nourrira souvent les programmes des listes d'Union de la Gauche lors des élections municipales de 1977 militant pour le renforcement des réseaux de bus et la réintroduction du tramway. Elle se cristallise peu à peu en doctrine au travers des réseaux professionnels que sont la Fédération nationale des agences d'urbanisme (FNAU, créée en 1980) et le Groupement des autorités responsables de transport (GART, créé la même année) qui assurent la diffusion de nouvelles manières d'envisager et d'organiser les mobilités urbaines.

La décentralisation entérine et renforce ce processus d'horizontalisation des circulations. Les réformes qui confient aux communes de nouvelles compétences s'opèrent sur fond de crise des modèles d'action et de l'expertise de l'État. Les services déconcentrés peinent à redéfinir leur rôle dans la fabrique locale des politiques urbaines. Par ailleurs, les nouvelles facilités de recrutement offertes aux villes privent l'État du monopole de recrutement des professionnels de l'urbain les mieux formés (Biland, 2012). Un pôle d'expertise urbain concurrent se structure ainsi peu à peu au sein des administrations municipales, intercommunales et de leurs satellites techniques à partir de projets et de politiques que les villes ont mené sans, voire contre, l'expertise étatique. Sur cette base, des modèles ne procédant plus d'une expertise d'État mais de la capitalisation d'expérimentations locales commencent à circuler en matière de planification (Rennes, Lyon) ou de déplacements (Grenoble, Nantes). La circulation des modèles entre villes s'en trouve bouleversée. Elle n'est plus descendante et fondée sur la standardisation mais davantage horizontale et fondée sur la réplication d'expériences locales. Toutefois, l'État ne disparaît pas complètement de la scène puisque ces circulations d'un nouveau type privilégient des canaux qui lui sont en réalité très liés. En effet, si les directions les plus « techniciennes " du ministère de l'Équipement sont peu impliquées dans la circulation des modèles inventés localement, des directions plus dominées 
trouvent dans la promotion des initiatives locales un moyen d'acquérir une légitimité au sein du ministère. C'est le cas de la direction de l'Architecture et de l'Urbanisme (DAU) qui, dirigée de 1989 à 1994 par Jean Frébault, ingénieur des Ponts et Chaussées et successivement directeur des agences d'urbanisme de Toulouse (1971-78) et de Lyon (1978-89), œuvre à la valorisation des expériences locales, notamment lyonnaises, qui participent à la relance de la planification urbaine (Frébault, Lemosquet, 1993). Ce sera ensuite le cas de l'atelier Projet urbain rattaché aux directions du ministère en charge de l'Urbanisme et animé depuis 1993 par Ariella Masboungi, architecte-urbaniste en chef de l'État, dont la raison d'être est la valorisation de bonnes pratiques en matière de composition urbaine à travers l'organisation de voyages d'études et la publication de monographies de projets locaux. Le Club Ville Aménagement, fondé en 1993 à l'initiative de Jean Frébault, Jean-Paul Baïetto, alors directeur de la SEM en charge du projet Euralille, et de François Ascher, professeur d'urbanisme, joue un rôle similaire. En partenariat avec l'administration du ministère en charge de l'urbanisme (qui y bénéficie de 5 sièges de "membres de droit»), il réunit des représentants de sociétés d'économie mixte et de sociétés publiques locales d'aménagement, d'établissements publics d'aménagement, d'offices publics HLM et de directions de l'aménagement de grandes intercommunalités urbaines. À l'image de la FNAU, le Club dispose de liens évidents avec l'État. II compte parmi ses membres des représentants de structures héritées des grandes heures de l'État aménageur (le réseau des SEM), de structures qui sont formellement des bras armés de l'État (les EPA) mais aussi de représentants du corps des IPC et de sa structure de réflexion et de gestion des carrières, le Conseil général de l'environnement et du développement durable (ex-Conseil général des Ponts et Chaussées). Mais, comme la FNAU et l'atelier Projet urbain, le Club Ville Aménagement n'est pas une structure de diffusion descendante d'une pensée, de modèles et de pratiques conçus in abstracto par une administration centrale et ses grands corps mais bien davantage un outil de capitalisation, de valorisation et de diffusion de bonnes pratiques inventées dans le cadre d'expériences locales. Ces canaux de diffusion sont dominés par des acteurs dont la légitimité ne procède pas tant de leur appartenance à l'administration centrale et ses grands corps que de leurs faits d'arme en tant que protagonistes d'expériences ou d'innovations locales.

Ces dynamiques d'horizontalisation des circulations vont prendre une nouvelle dimension avec le développement de la politique régionale et urbaine de l'Union européenne. II est désormais établi que la construction européenne a impacté de manière significative le gouvernement des villes et les relations entre les villes et les États. "Tout à coup, indique Le Galès, le face-à-face ancien entre État et villes, entre centre et périphérie, devient pour une part obsolète. Le terrain de jeu politique a changé d'échelle, le nombre de joueurs s'est multiplié et, surtout, aucun joueur ne détient plus le monopole de la production des règles ni de la contrainte » (Le Galès, 2011, p. 172). Ni de l'expertise pourrait-on ajouter. Si la construction européenne correspond à l'émergence d'une nouvelle source de contrainte (sous la forme notamment de transferts directs contraints pour reprendre la typologie de Marsh et Dolowitz [2000]), elle va aussi ouvrir de nombreux espaces propices à des transferts volontaires. En ce qui concerne les villes françaises, ce ne sont pas tant les politiques urbaines de l'Union européenne qui vont être source d'inspiration. Des programmes comme les projets pilotes urbains (PPU) ou Urban I et Urban II, qui s'inspirent largement des politiques françaises et britanniques en direction des quartiers en difficulté, vont avoir davantage 
d'impact dans les pays dans lesquels ce type de politique publique n'existait pas (Pasquier, Pinson, 2004). Ce sont surtout les nombreux réseaux et associations de villes qui vont permettre aux élus et techniciens des villes françaises à la fois de prendre connaissance de nouveaux modèles et de les importer mais aussi (et peut-être surtout) de promouvoir leurs propres pratiques (Payre, 2010). La relance des politiques régionales de l'Union européenne à partir de la fin des années 1980 va en effet s'accompagner d'une prolifération des réseaux de villes transnationaux (Benington, Harvey, 1999). Ces réseaux se structurent tantôt directement autour de programmes européens, tantôt autour de spécificités urbaines (spécialisations économiques, problématiques sociales ou géographiques), d'autres encore ont une vocation généraliste, comme le réseau Eurocités. Ces réseaux ont plusieurs intérêts pour les villes qui y participent. Ils permettent de faire entendre leur voix au sein des instances européennes. Ils sont aussi des outils de veille sur l'activité réglementaire européenne. Enfin et surtout, ils constituent des espaces privilégiés d'échanges d'expériences et d'apprentissage pour les représentants des villes. L'intensité des échanges au sein de ces réseaux donne à voir l'espace européen non pas comme un ensemble d'institutions surplombantes agissant essentiellement au travers de l'imposition descendante des changements réglementaires, mais comme une vaste plate-forme de transfert ("massive transfer platform ", Radaelli, 2000, p. 26). Cette situation est encore plus nette pour les villes depuis que les programmes urbains de l'Europe finançant des actions en matière d'aménagement urbain et de développement social (PPU, Urban) ont laissé la place en 2007 à un programme, Urbact, essentiellement orienté vers le transfert d'expertise entre les villes.

\section{Verticalisation : l'État régulateur}

La mise en perspective historique rappelle avec force que l'importance des circulations horizontales dans les politiques urbaines est intimement - et inversement - liée à l'emprise étatique sur la conception et la mise en œuvre de ces politiques. En cela, l'essor récent des circulations entre villes constitue tout à la fois une manifestation et un levier d'un processus de « desserrement du verrou de l'État » (Le Galès, 1999) dont les ressorts sont suffisamment connus pour qu'il ne soit pas nécessaire d'y revenir longuement. La décentralisation, la déconcentration qui l'a prolongée, la montée en puissance de pouvoirs régionaux et métropolitains, l'européanisation des politiques publiques, la globalisation économique, le rôle croissant des acteurs privés dans l'action publique, l'émergence de problèmes socio-économiques et environnementaux complexes irréductibles aux découpages sectoriels et aux solutions standardisées des administrations se sont combinés pour favoriser l'autonomisation des villes vis-à-vis de la tutelle étatique.

Le lien ainsi établi entre intensification des circulations horizontales et réduction de l'emprise verticale de l'État sur les politiques urbaines procède pour l'essentiel d'analyses empiriques développées dans les deux décennies qui ont suivi la décentralisation. Les réformes institutionnelles de la première décennie des années 2000 invitent à réinterroger ce lien. Si elles ont renforcé l'autonomie des villes et apparaissent donc favorables aux transferts horizontaux, elles ont aussi permis à l'État de retrouver des capacités d'orientation de leur action, qui procèdent notamment de son investissement dans l'animation des transferts interurbains appuyée sur une activité intense de sélection, de modélisation et de promotion des "bonnes pratiques". Privé des ressources d'expertise et d'autorité qui lui 
permettaient d'imposer aux villes ses priorités et ses solutions, l'État bénéficie en effet encore d'importantes ressources de légitimité qui lui permettent, en distinguant des villes et des expériences modèles, d'orienter les choix des villes importatrices. La perte de centralité de l'État dans les politiques urbaines a ainsi pu être compensée par son repositionnement plus nodal dans ces transferts, conduisant à l'introduction d'une dimension verticale dans des circulations qui apparaissent de prime abord purement horizontales.

Considérons de façon plus détaillée les transformations du contexte institutionnel dans lequel cette verticalisation des transferts interurbains a pris corps. Avec l'Acte $\|$ de la décentralisation de 2003-2004 et les diverses lois réformant l'architecture institutionnelle des territoires ${ }^{3}$, les villes se sont vues confier de nombreuses compétences qu'elles partageaient avec l'État. Le " décroisement des compétences » (Le Lidec, 2007) a ainsi mis fin à un régime de cogestion des politiques urbaines issu de la décentralisation des années 1980 et de la déconcentration de la décennie suivante. Disposant de l'entière responsabilité de nombreuses politiques publiques qui faisaient jusqu'alors l'objet de négociations et de contractualisations avec les services déconcentrés de l'État, les villes ont intensifié leurs échanges pour trouver chez leurs homologues les instruments, les modes opératoires et l'expertise qui n'étaient plus fournies par un État local en voie de résidualisation. Car l'approfondissement de la décentralisation ne s'est pas accompagné d'un surcroît de déconcentration. Au contraire, les réformes de l'État menées en parallèle ou à la suite de l'Acte II de la décentralisation ont organisé un mouvement de re-concentration, du niveau départemental vers le niveau régional et de ces deux niveaux vers le niveau central. De la loi organique relative aux lois de finances du $1^{\text {er }}$ août 2001 (LOLF) aux mesures de la Révision générale des politiques publiques (RGPP), en passant par la multiplication d'agences chargées de la mise en œuvre des programmes étatiques, ces réformes de l'État d'inspiration néomanagériale ont profondément renouvelé les modalités d'énonciation des politiques publiques, redistribué les responsabilités entre acteurs, et transformé l'organisation et les instruments de l'État. L'instrumentation performantielle de la LOLF a conduit à une nette verticalisation des politiques étatiques, réduisant les marges de manœuvres des services déconcentrés pour organiser la territorialisation des programmes nationaux (Epstein, 2013a; Debar, 2013). Combinée avec l'Acte II de la décentralisation qui avait fait perdre à ces derniers d'importantes ressources humaines et financières, la LOLF a ensuite ouvert la voie à une réforme de l'administration territoriale de l'État dans le cadre de la RGPP qui, en organisant de vastes et complexes fusions des services déconcentrés et en donnant la prééminence au niveau régional (Poupeau, 2013), a distendu les relations de ces services avec ceux des villes.

En même temps qu'elles ont renforcé l'autonomie des villes vis-à-vis d'un État qui s'est retiré de la cogestion des politiques urbaines, les réformes institutionnelles de la dernière décennie ont permis à l'État, directement ou via ses agences, de retrouver des capacités de contrôle et d'orientation à distance des politiques développées par les pouvoirs urbains. En introduisant dans les administrations des logiques d'action, des instruments d'intervention et des formats organisationnels inspirés par les préceptes du New Public Management,

3. Lois du 12 juillet 1999 relative au renforcement et à la simplification de la coopération intercommunale, du 16 décembre 2010 portant réforme des collectivités territoriales, du 27 janvier 2014 de la modernisation de l'action publique territoriale et d'affirmation des métropoles, et du 6 août 2015 portant nouvelle organisation territoriale de la République (dite loi NOTRe). 
ces réformes ont transformé l'intervention de l'État. D'un côté, elles ont organisé son retrait progressif de la mise en œuvre de ses propres politiques, pour passer du faire au faire-faire ; de l'autre côté, elles lui ont permis de se doter de nouveaux instruments de pilotage de l'action conduite par des tiers. Elles ont donc donné lieu à deux évolutions interdépendantes de son rôle dans l'action publique, dont la notion d'État régulateur - entendu ici comme « un État qui agit plus indirectement que directement, qui est plus en interaction qu'en action, qui délègue plus qu'il n'intervient directement, qui pilote et qui oriente plus qu'il ne met en œuvre [...] à travers le développement de l'audit, de l'évaluation, du benchmarking, du contrôle de qualité, etc. » (Hassenteufel, 2008, p. 326) - permet de rendre compte.

Les labels et prix par lesquels l'État distingue publiquement une ville ou un projet constituent, au même titre que l'audit ou le benchmarking, des instruments privilégiés de l'intervention territoriale de cet État régulateur. N'ayant plus l'autorité et les ressources financières pour imposer ses priorités et ses solutions aux villes, ni même souvent l'expertise suffisante pour les définir, l'État recourt désormais intensivement à ces instruments incitatifs appuyés sur des mécanismes compétitifs pour peser à distance sur les agendas urbains et sur les circulations interurbaines. Si l'essor de ce " gouvernement par les labels " (Bergeron et al., 2014) peut s'observer dans de nombreux secteurs, il concerne plus particulièrement les domaines correspondant à des problématiques émergentes de l'action publique, marqués par une faible formalisation des problèmes et des solutions, une distribution incertaine des responsabilités entre diverses institutions et professions, ainsi que par un déficit de légitimité et de ressources financières. Des domaines tels que le développement durable (cf. infra), l'économie numérique (label métropole FrenchTech), ou encore la lutte contre les discriminations (label diversité) rentrent clairement dans cette catégorie.

Qu'elles viennent certifier le respect de normes allant au-delà des exigences réglementaires ou qu'elles distinguent l'exceptionnalité, ces récompenses attribuées (ou patronnées) par l'État incitent les villes à se dépasser, à innover pour respecter les critères de labellisation ou pour faire mieux que les autres villes. Ainsi, à l'image des pratiques de benchmarking, les récompenses étatiques enrôlent les villes « dans une course sans ligne d'arrivée [...], car il s'agit d'une mission d'amélioration continue de la qualité, un effort sans relâche pour faire mieux, une tension constante pour être le meilleur » (Bruno, Didier, 2013, p. 87). Même lorsque les rétributions sont purement symboliques, elles ne sont pas sans valeur pour les villes, qui les utilisent comme des signes de distinction permettant de valoriser leur territoire dans la compétition interurbaine ${ }^{4}$. Les effets de ces instruments dépassent les seules villes qui entrent dans le jeu compétitif pour l'accès à la reconnaissance étatique. Les trophées et labels fonctionnent en effet comme des dispositifs de certification dont s'alimentent les transferts d'action publique (Ancelovici, Jenson, 2012). En distinguant une ou plusieurs villes, l'État les érige en modèles pour toutes les autres, leur fournissant non seulement un étalon de benchmarking à l'aune duquel elles peuvent s'autoévaluer mais aussi des répertoires de «bonnes pratiques » pour se mettre à niveau. À l'image de la légion d'honneur analysée par

4. Ainsi, dans le cas du label FrenchTech, les métropoles labellisées ne bénéficient pas directement des maigres budgets consacrés par l'État à cette politique de développement de l'économie numérique, qui sont dévolus à la promotion internationale de la marque FrenchTech et au financement d'accélérateurs et de start-ups sans prise en compte de leur localisation. Toutefois, ces métropoles gagnent le droit d'utiliser cette marque dans leur communication, et peuvent donc en tirer des bénéfices d'image. 
Olivier Ihl (2007), ces récompenses dont l'attribution opère une distinction parmi des égaux en érigeant en modèles ceux qui ont fait la preuve de leurs mérites, permettent à l'État de " conduire les conduites " (Foucault, 1975) autonomes.

\section{Piloter à distance les politiques urbaines : uploading et downloading}

Dans cette deuxième partie, au contenu plus nettement empirique, nous voudrions étayer le cadre d'analyse esquissé dans la partie précédente en montrant comment, concrètement, les logiques d'horizontalisation des circulations entre villes d'une part et de "retour de l'État " dans un rôle de promoteur des échanges et de certificateur des bonnes pratiques d'autre part s'articulent et se complètent. Nous le faisons au travers de l'exemple de deux programmes spécifiques : le Programme national de rénovation urbaine (PNRU) et le plan Ville durable. Dans les deux cas, l'État a délégué aux villes la responsabilité de concevoir et mettre en œuvre des projets assurant la déclinaison d'objectifs qu'il a tracés à grands traits mais que les initiatives locales ont vocation à préciser et opérationnaliser. Dans les deux cas, les projets des villes s'alimentent d'une activité intense d'échanges et de circulations interurbains. Même si elles peuvent parfois déborder l'espace national, ces circulations s'effectuent essentiellement dans un cadre national et sont en grande partie organisées par l'État. Ce que l'on observe au final relève autant de mécanismes d'uploading (faire remonter des pratiques, les sélectionner et les modéliser) que de downloading (diffuser ces «bonnes pratiques ", les intégrer aux cadres de l'action publique). Ces mécanismes permettent à l'État de retrouver des capacités de mobilisation et d'orientation à distance des politiques menées localement.

\section{Le PNRU : les transferts horizontaux comme levier de pilotage vertical}

Lancé en 2003 par Jean-Louis Borloo, le Programme national de rénovation urbaine prévoit l'investissement de 45 milliards d'euros pour organiser la démolition-reconstruction de 490 quartiers, dont 12 milliards d'euros proviennent de subventions allouées par l'Agence nationale de la rénovation urbaine (ANRU). Parce qu'il s'agit, suivant l'expression prisée par ses promoteurs, du " plus grand programme civil de l'histoire française ", et parce qu'il prend pour cibles des grands ensembles d'habitat social aussi emblématiques des politiques urbaines des années 1960-1970 que de leurs transformations au cours des deux décennies suivantes, le PNRU fournit un éclairage important sur les évolutions récentes de l'action publique. Son examen donne tout d'abord à voir un double mouvement d'autonomisation des villes vis-à-vis de l'État d'un côté, de recomposition de ce dernier et de renouvellement de ses capacités d'orientation des politiques définies et mises en œuvre par les villes d'un autre côté. II permet ensuite de saisir les modalités de ce pilotage vertical, qui procède de la mise en concurrence des villes par le biais d'appels à projet et de la mise en circulation de leurs «bonnes pratiques". L'ANRU fournit ainsi une illustration épurée de la figure de l'État régulateur orchestrant la sélection et la circulation des modèles d'action.

Les quartiers de grands ensembles visés par le PNRU sont de parfaits symboles des politiques urbaines des Trente Glorieuses. La procédure des zones à urbaniser par priorité 
(ZUP) qui leur a donné naissance conférait à l'État la maîtrise totale de leur production. Créées par décret gouvernemental sur la base d'études préparatoires des administrations étatiques (ministère de la Reconstruction et du Logement, puis ministère de l'Équipement), leur construction a été pilotée par des filiales de la CDC qui ont organisé la production massive d'immeubles et d'équipements collectifs, déclinant dans toutes les villes françaises un même modèle national formalisé dans la fameuse "grille Dupont ", qui déterminait précisément le contenu des programmes opérationnels des ZUP (Merlin, 2010).

La politique de la Ville (initialement dénommée politique de développement social des quartiers) qui a émergé au début des années 1980 en réponse aux manifestations de la crise socio-urbaine dans ces quartiers, procède d'une toute autre approche. Dans un contexte de décentralisation, elle tourne le dos à la logique descendante et standardisatrice de l'État aménageur, pour faire primer une logique de projet territorial et de négociation contractuelle entre collectivités et services déconcentrés (Donzelot, Estebe, 1994 ; Maillard, 2004). Cette approche et les instruments d'action publique qu'elle a introduits s'étant rapidement diffusés dans de nombreuses politiques urbaines, la politique de la ville peut être vue comme fondatrice d'un nouveau modèle de conduite de ces politiques, dans le double sens du prototype (expérimentation) et de l'idéal-type (exemplarité). Dans ce modèle, l'État n'impose plus aux villes ses solutions mais les incite à en inventer de nouvelles, cette incitation s'appuyant notamment sur la capitalisation et la valorisation des expérimentations locales ${ }^{5}$. Pour les administrations nationales en charge de la politique de la Ville dans les années 1980 et 1990 (Commission nationale pour le développement social des quartiers puis Délégation interministérielle à la Ville), il s'agissait alors surtout de donner à voir la fertilité d'une approche expérimentale et remontante, et non de mettre en avant de manière sélective une poignée d'expériences locales érigées en modèles.

La loi d'orientation et de programmation pour la ville et la rénovation urbaine du $1^{\text {er }}$ août 2003 (dite loi Borloo) a considérablement accru les moyens dévolus à la politique de la Ville, en imposant au $1 \%$ logement $^{6}$ de verser près d'un demi-milliard d'euros par an à l'ANRU. Elle a aussi transformé le design institutionnel de cette politique pour la mettre en conformité avec l'Acte II de la décentralisation qui était alors en préparation et avec la LOLF votée en 2001 mais dont la pleine entrée en application était prévue en 2006. À l'approche transversale et remontante promue jusqu'alors, s'est substitué un ensemble de programmes sectoriels organisés autour d'objectifs quantifiés et d'indicateurs de performance, portés par des agences nationales autonomes ${ }^{7}$ (Epstein, 2013b). Cette réforme justifiée sur les registres de l'autonomie et des responsabilités locales, de la simplification administrative et de la performance gestionnaire, devait permettre aux villes de s'émanciper des procédures contractuelles de l'État local et d'alléger les contraintes que faisaient peser sur leurs projets

5. La base documentaire en ligne du ministère de la Ville (i.ville.gouv.fr) compte ainsi plus de 900 fiches d'expérience et s'enrichit de 40 à 100 nouvelles fiches par an.

6. Le «1\% logement " (rebaptisé «Action logement " en 2009) est un dispositif paritaire de gestion de la Participation des employeurs à l'effort de construction (PEEC), instituée en 1953 pour les entreprises du secteur privé non agricole. À l'origine, celles-ci devaient consacrer $1 \%$ de leur masse salariale au financement de la construction de logements. Depuis 1992, ce taux est fixé à 0,45 \% de la masse salariale de l'année précédente.

7. La création de I'ANRU a été suivie, en 2006, par celle de l'Agence nationale pour la cohésion sociale et l'égalité des chances (ACSE) chargée du pilotage des programmes sociaux et éducatifs de la politique de la ville. 
leurs multiples bailleurs de fonds. L'ANRU fonctionne en effet comme un guichet unique pour des projets de démolition-reconstruction librement définis par les villes et mis en œuvre sous la seule responsabilité des maires. Son action dirigée vers des quartiers construits par l'État puis cogérés avec les acteurs locaux vient donc parachever le processus d'autonomisation des villes, d'autant plus que l'ANRU est elle-même une agence autonome, dont les orientations sont définies par un conseil d'administration dans lequel les représentants des ministères n'occupent que la moitié des sièges ${ }^{9}$.

Mais en même temps qu'elle a accru l'autonomie des villes, la loi Borloo a permis à l'État de retrouver des capacités d'orientation et de contrôle des politiques développées par celles-ci, qui s'étaient largement dissolues dans les politiques «partenariales " des deux décennies post-décentralisation. Ce mouvement paradoxal résulte d'abord de l'appel à projet utilisé par l'ANRU pour sélectionner les villes bénéficiant de ses subsides. Le recours à cet instrument apparait cohérent avec les principes d'autonomie, de subsidiarité et de performance mis en avant par le législateur : les projets sont élaborés à l'initiative des acteurs locaux, sans interférence de l'État, et la mise en concurrence des villes pour l'accès aux crédits nationaux est supposée favoriser l'émulation et l'innovation. Mais le recours à un appel à projet a surtout eu pour effet d'inciter les villes à se conformer aux attentes nationales (Breton, 2014). Les montants en jeu et l'incertitude sur les résultats de la compétition les a poussées à élaborer des projets déclinant fidèlement les objectifs stratégiques et opérationnels de l'ANRU, qui attendait notamment qu'elles conduisent des démolitions massives de logements sociaux. Les capacités d'engagement budgétaire du guichet unique se réduisant progressivement, à mesure de la signature de conventions avec les villes sélectionnées, son soutien est devenu de plus en plus parcimonieux et exigeant. L'appel à projet s'est alors transformé en course de vitesse entre des villes soucieuses de voir leur projet retenu au plus tôt, et prêtes pour ce faire à intégrer - voire à anticiper - les attentes de l'agence (Epstein, 2013b). La mise en concurrence des villes a ainsi permis à l'État de peser sur les agendas urbains et sur le contenu des projets des villes, comme en témoigne la prolifération des projets de démolition-reconstruction déclinant fidèlement la doctrine urbanistique de l'agence nationale dans des centaines de villes, dont la majorité n'avait jamais envisagé de telles opérations mobilisant une part substantielle de leurs budgets d'investissement.

Dans un contexte de resserrement des marges de manœuvres budgétaires des gouvernements nationaux et locaux, l'allocation concurrentielle de milliards d'euros a incité les villes à se conformer, en toute liberté, aux attentes nationales. Elles l'ont fait d'autant plus aisément que ces attentes n'ont jamais été présentées comme des prescriptions étatiques, mais comme le produit de la capitalisation des expériences locales. Le contenu attendu des projets de rénovation urbaine n'était pas précisément défini au lancement du PNRU. II a été progressivement explicité par l'ANRU, à mesure de l'examen des projets qui lui étaient

8. L'ANRU mutualise l'ensemble des budgets nationaux dévolus aux projets d'aménagement dans les quartiers prioritaires de la politique de la ville, qui étaient auparavant distribués par les ministères de l'Équipement et de la Ville, les diverses structures du 1 \% logement, la Caisse des dépôts et consignations ainsi que la Caisse de garantie du logement locatif social.

9. Notons que l'ANRU échappe ainsi à la tutelle du ministère de l'Équipement, mais qu'elle demeure sous l'emprise du grand corps de ce ministère : son premier président était un ingénieur des Ponts et Chaussées, tout comme les directeurs généraux qui se sont succédé à sa tête jusqu'en 2014 ainsi que la grande majorité de leurs adjoints. 
soumis par les villes. Cette explicitation s'est formalisée dans le règlement financier de l'agence, qui détermine les types d'opérations éligibles à son soutien (assiette) et le niveau relatif de ce soutien (taux), autrement dit, le possible et le souhaité. Ce règlement s'est en effet construit sur la base des projets proposés par les villes, dans une logique jurisprudentielle qui a conduit à transcrire en règles générales (opérations éligibles et taux de subvention) des décisions prises à l'occasion de l'examen d'un projet singulier.

Tout autant que sur la mise en concurrence des villes, le pilotage national de la rénovation urbaine s'est appuyé sur la mise en circulation de leurs expériences. L'ANRU a développé une activité intense de communication fondée sur la valorisation des premiers projets qu'elle a soutenus et des «bonnes pratiques » qui y ont été développées. Elle a aussi structuré des espaces et des réseaux d'échanges, dans lesquels les projets des villes pionnières étaient systématiquement mis en avant. Cet investissement dans l'animation des échanges interurbains était d'autant plus nécessaire que des centaines de communes de banlieue et de villes moyennes candidates au PNRU ne disposaient en interne ni de l'expérience, ni des compétences nécessaires pour concevoir puis conduire des projets urbains complexes comme le sont les projets de rénovation urbaine. Plutôt que de s'appuyer sur les services déconcentrés de l'État pour aider ces villes à formaliser et mettre en œuvre leur projet, l'ANRU les a incitées à se faire assister par des prestataires privés, en prenant en charge jusqu'à $50 \%$ de leurs honoraires. Les urbanistes-conseils et les multiples consultants missionnés pour assister les maîtrises d'ouvrages des projets de rénovation urbaine (conseils en organisation et en gestion de projet, programmistes, professionnels de la communication, etc.) ont été les principaux vecteurs des circulations entre villes engagées dans le PNRU, en même temps que les meilleurs promoteurs des attentes de l'ANRU en leur direction. Certains d'entre eux ont bénéficié d'un soutien particulièrement soutenu de l'agence, qui leur a permis d'acquérir une visibilité nationale et, en multipliant les contrats, de colporter de ville en ville des approches, des solutions techniques et des modes opératoires d'autant plus aisément adoptés qu'ils n'apparaissaient pas comme des prescriptions étatiques mais comme le produit d'expériences conduites ailleurs. Ces consultants et les projets locaux qu'ils ont accompagné ont été mis en avant par l'ANRU dans ses multiples documents de communication ${ }^{10}$ ou à l'occasion des congrès nationaux et des forums inter-régionaux des acteurs de la rénovation urbaine qu'elle organise chaque année. L'investissement de l'ANRU dans la structuration de ce milieu professionnel s'est même concrétisé en 2005 par la constitution de plusieurs "pôles ressources " réunissant certains de ces prestataires, qui interviennent à l'initiative et aux frais de l'agence pour aider les villes qui peinent à répondre à ses attentes ${ }^{11}$.

Le pilotage national des opérations d'import/export entre villes du PNRU s'est aussi appuyé sur l'élaboration de guides de « bonnes pratiques » issues du recensement de « réussites locales » en matière de développement économique des quartiers en rénovation urbaine

10. Cf. le numéro hors-série de la revue Urbanisme publié en 2007 à l'initiative de l'ANRU («Rénovation urbaine : enjeux, mise en œuvre, qualités "), qui consacre une large place aux interviews de ces professionnels.

11. Le premier de ces pôles, intitulé "qualité urbaine ", est composé d'une quinzaine d'architectes et d'urbanistes, dont de nombreux grands noms de la profession (Roland Castro, Bruno Fortier, Jean-Patrick Fortin, Antoine Grumbach, Philippe Panerai, etc.). II a été suivi par un pôle consacré au développement économique, un pôle concertation, un pôle d'experts de la conduite de projet, et un pôle chargé de préparer les audits à mi-parcours et les évaluations finales. 
ou de préparation de la sortie du projet, ainsi que sur l'organisation de concours et l'attribution de prix distinguant les villes modèles en matière de "rénovation urbaine et urbanisme durables " et de "qualité urbaine, architecturale et paysagère ". L'objectif poursuivi était alors, suivant la présentation qui est faite du premier de ces prix sur le site internet de l'ANRU, de "repérer et diffuser les savoir-faire ainsi que les bonnes pratiques en matière de développement durable ", de "valoriser les démarches des précurseurs " et de "nourrir les échanges du réseau des acteurs de la rénovation urbaine afin d'amplifier les dynamiques innovantes de terrain et progresser dans ce domaine ". 57 villes ont postulé au premier et 98 au second, cette participation massive au concours s'expliquant moins par la valeur monétaire des récompenses (100 000 euros de crédits d'ingénierie) que par leur valeur honorifique : la remise des prix a lieu lors des Journées nationales d'échanges de la rénovation urbaine, grand raout annuel réunissant l'ensemble des acteurs du PNRU devant lesquels les projets distingués sont érigés en modèles.

Trois enseignements peuvent être tirés de cette analyse du PNRU. Premièrement, ce programme et sa mise en perspective historique permettent de saisir la dynamique de long terme qui a vu l'État aménageur se transformer en un État régulateur. La délégation aux villes de l'entière responsabilité de la conception et de la mise en œuvre des projets de rénovation urbaine ne marque cependant pas la disparition de l'emprise étatique sur les projets menés dans les grands ensembles. Au contraire, le renforcement de l'autonomie des villes permet à un État recomposé de piloter à distance ces grands projets urbains. Ce pilotage central procède d'abord d'une mise en concurrence des villes, qui les incite à se conformer aux attentes de l'agence nationale. II s'appuie aussi, c'est le deuxième enseignement de cette étude, sur la valorisation sélective de projets (ou de porteurs de projets) qui sont mis en avant par cette agence. En contrôlant les contenus mis en circulation dans les réseaux d'échanges entre acteurs qu'elle anime, l'agence peut utiliser les transferts horizontaux entre villes comme des leviers de pilotage vertical. Troisièmement, si le PNRU donne lieu à d'intenses activités d'import/export entre villes, ces échanges s'opèrent dans un cadre purement national. Alors même que plusieurs pays voisins conduisent des politiques de régénération urbaine de leurs quartiers d'habitat social et que de nombreuses villes françaises participent à des réseaux de villes européens ou ont accès au programme d'échange d'expériences Urbact, les expériences des villes britanniques, hollandaises ou allemandes demeurent méconnues des acteurs de la rénovation urbaine, qui puisent leur inspiration dans des expériences plus proches, promues dans les cadres institués par l'ANRU.

\section{Le plan Ville Durable : construire l'action de l'État à partir de projets locaux exemplaires}

Les politiques de développement durable partagent de nombreux points communs avec la politique de rénovation urbaine. Depuis le milieu des années 2000, elles font en effet la part belle aux instruments néomanagériaux, comme les appels à projet compétitifs, qui ont permis à l'État de renforcer son emprise sur les villes dans un domaine où il n'avait jamais disposé d'une forte capacité d'intervention (Béal, 2011). Toutefois, malgré ces similitudes avec la rénovation urbaine, les politiques de développement durable s'en démarquent sur plusieurs points. Tout d'abord, dans le domaine flou du développement durable, l'État n'a pas été en mesure de prescrire des contenus précis de politiques publiques qu'il aurait ensuite imposés 
par le biais d'un appel à projet. Ici, l'outil appel à projet sert davantage à faire émerger ces contenus par l'identification de modèles qui seront diffusés dans un second temps. Deuxièmement, les politiques de développement durable ne sont pas dotées d'une enveloppe financière comparable à celle du PNRU. Elles s'appuient davantage sur des ressorts " doux " de gouvernement et sur une gestion symbolique des territoires au travers de labels, de prix ou encore de mises en réseaux. Enfin, ces politiques, qui sont pensées au départ dans un contexte national, prennent également sens dans un souci de renforcer l'influence de la France à l'international en exportant certains savoir-faire dans les pays des Suds.

Le plan Ville durable, lancé en 2008 par le ministère de l'Écologie, du Développement et de l'Aménagement durables, est la première tentative d'élaboration d'un programme structurant à destination des villes dans les domaines de l'environnement et du développement durable. Auparavant, le rôle de l'État dans les politiques territoriales de développement durable était particulièrement limité. À l'exception de quelques appels à projet (faiblement compétitifs) sur les agendas 21 locaux au tournant des années 2000, l'État avait laissé une grande marge de manœuvre aux acteurs locaux, qui pouvaient s'appuyer sur de multiples réseaux de villes (ICLEl, Énergie-Cité, Campagne européenne des villes durables, etc.) et programmes européens (SAVE, LIFE, Concerto, etc.). C'est donc avec la tenue du Grenelle de l'Environnement et la création d'un "super-ministère " du Développement durable que l'action de l'État à destination des villes se réorganise. En effet, en 2008, le plan Ville durable est lancé dans l'objectif de « favoriser l'émergence d'une nouvelle façon de concevoir, construire, faire évoluer et gérer la ville» (MEDDTL, 2008). Ce plan comporte au départ trois volets - l'appel à projet ÉcoQuartiers, la démarche ÉcoCités et l'appel à projet « Transports collectifs en site propre »- qui seront complétés un an plus tard par le plan « Restaurer et valoriser la nature en ville ». En son sein, l'appel à projet ÉcoQuartiers et la démarche ÉcoCités méritent une attention particulière en raison de leur rôle dans les circulations verticales et horizontales de « modèles » de politiques urbaines.

Lancé dès 2008, l'appel à projet ÉcoQuartiers ne comportait pas de prescriptions claires à destination des villes. Si les acteurs locaux devaient respecter certains objectifs généraux (formes urbaines denses, performance énergétique des bâtiments, modes de transport doux, démarche partenariale, etc.), ils disposaient d'une marge de manœuvre pour construire leurs candidatures. L'absence de financement attribué aux lauréats et la volonté de valoriser des démarches déjà engagées rendaient difficile l'imposition de contenus précis qu'auraient dû respecter les villes. Ainsi, l'objectif de cet appel à projet résidait davantage dans la mise en réseau d'acteurs, permettant de faire remonter des expériences " exemplaires » sur la base desquelles un référentiel, puis un label pourraient être construits. Censé se limiter aux 28 lauréats, le Club national ÉcoQuartiers mis en place en 2010 est finalement ouvert à l'ensemble des 150 candidats, notamment par la création d'antennes régionales. Regroupant des représentants des collectivités, de l'État et des milieux de l'aménagement, ce club a joué un rôle central dans la définition d'un référentiel, qui a servi d'étalon pour sélectionner les lauréats du second concours lancé fin 2011. Animé par la direction de l'Habitat, de l'Urbanisme et des Paysages (DHUP), il s'est également progressivement institutionnalisé autour d'activités de diffusion de "bonnes pratiques " (notamment, par le biais de conférences et de publications), de formation des agents des collectivités et de production d'un label, construit par la capitalisation des démarches considérées comme étant innovantes et exemplaires. 
Dans la foulée de la nomination des 24 nouveaux lauréats en 2012, le label national ÉcoQuartier est mis en place. II permet aux collectivités - ayant au préalable signé la charte ÉcoQuartiers et intégré la démarche nationale - de faire une demande de labellisation ${ }^{12}$ dont l'octroi repose sur la validation de plusieurs critères : 20 critères d'évaluation (liés aux enjeux « locaux ») et 20 indicateurs chiffrés (liés aux enjeux "globaux»). L'objectif de ce label est triple. II s'agit tout d'abord de favoriser l'émergence d'un cadre (certes flexible) permettant de répondre aux engagements internationaux de l'État (le Paquet énergie-climat de l'Union européenne, le Protocole de Nagoya, etc.) et sur lequel les acteurs locaux, les agences (ADEME) et les représentants de l'État (DREAL), peuvent s'appuyer pour construire les futurs projets d'écoquartiers. Ainsi, en faisant remonter des expériences innovantes, l'État reconstruit une expertise sur les questions de développement durable et une capacité à généraliser la démarche ÉcoQuartiers à un nombre important de villes ${ }^{13}$. II s'agit ensuite de lutter contre la réappropriation généralisée du terme "écoquartier " et sa perte de sens. Selon les mots de la ministre, "le label sera un gage d'exemplarité de la démarche, pour éviter l'accusation de greenwashing, ce côté vitrine qui a parfois fait du tort aux véritables opérations intégrées et réussies. Ce sera aussi une garantie de qualité » (Duflot, 2012). II s'agit enfin de valoriser les expériences françaises à l'international. Ainsi, les trois grands prix ÉcoQuartier - la ZAC de Bonne à Grenoble (2009), la ZAC de l'Union de Roubaix-Tourcoing-Wattrelos (2011), le Plateau de Haye de Nancy-LaxouMaxéville (2011) - ont été intégrés au benchmark européen réalisé dans le cadre de la mise en place du cadre européen de référence de la ville durable ${ }^{14}$. Cette intégration est pensée comme un moyen de rendre visibles ces démarches à l'international et de "vendre " l'approche française dans les pays où la production d'écoquartiers est encore balbutiante : "Le label ÉcoQuartier sera également un levier de valorisation, de comparaison et de partage à l'échelle internationale d'une vision de la ville durable. II sera l'occasion de diffuser les bonnes pratiques et les savoir-faire français. [...] Des premières présentations de la démarche française (à Varsovie, Rio, Naples, Cadix...) ont déjà montré la pertinence de l'approche et l'intérêt des pays étrangers pour une éventuelle adaptation dans leur contexte national » (METL, 2012, p. 12).

Si le concours ÉcoQuartiers n'était pas marqué par une logique de pilotage vertical, il en va tout autrement pour la démarche ÉcoCités. Lancée en 2009, elle vise la réalisation de " grands projets d'innovation architecturale, sociale et énergétique » dans les agglomérations de plus de 100000 habitants. Au départ, le flou entoure la question des moyens financiers

12. À ce jour, 32 opérations ont été labellisées par le ministère qui s'appuie dans son évaluation sur une commission nationale mêlant acteurs publics et privés.

13. Ce souci de mobilisation suscite des craintes chez certains acteurs locaux, comme le montre cet extrait d'un entretien réalisé auprès d'un technicien du CETE-Ouest : " L'État pousse pour qu'un label écoquartier soit mis en place, même si je pense qu'on s'en tiendra à un référentiel. C'est dommage de vouloir construire à l'avance ce que doit être un écoquartier. Nous, au CETE, on cherche à éviter qu'un modèle ne naisse, que les écoquartiers se résument à des bâtiments passifs, un tramway, des coulées vertes, etc. On veut que le contexte reste important, que les gens continuent à réfléchir [...]. Pour les A21L, le cadre de référence a été construit avec les collectivités territoriales. C'était beaucoup moins descendant que les écoquartiers. $\|$ y avait eu des assises régionales. Cela vient peut-être de la transformation du ministère. Avant, ils étaient moins nombreux, leur réseau territorial était moins structuré et du coup ils s'appuyaient peut-être plus sur les collectivités territoriales. Maintenant c'est plus directif. Quand le ministère dit "on va là-dessus", tout le monde charge... " (Entretien, 27 octobre 2009.)

14. Le RFSC (Reference Framework for Sustainable Cities) est un outil opérationnel créé par I'Union européenne en 2013 pour offrir aux villes un support d'expertise dans la réalisation de leurs démarches d'écoquartiers [http//www.rfsc-community.eu/]. 
alloués à cette démarche. Pourtant, la plupart des collectivités de taille importante, désireuses de se positionner sur l'enjeu émergent du développement durable, répondent à l'appel dont les critères de sélection - caractère innovant, sobriété énergétique, performance en termes de limitation des émissions de gaz à effet de serre, etc. - sont plus stricts que ceux du concours ÉcoQuartiers. Après l'annonce des 13 lauréats fin $2009^{15}$, la démarche change d'envergure en étant complétée par le programme Ville de demain qui cherche à faire émerger des " actions démonstratrices et exemplaires » susceptibles " d'être dupliquées » et d'influencer les pratiques d'aménagement durable aux échelles nationale et internationale (DALN, 2011, p. 1). Lancé à l'occasion du Grand Emprunt, ce programme signe l'émergence d'une vision plus coercitive des rapports villes/État autour des thématiques de développement durable (Béal, Pinson, 2015). Cette reprise en main est marquée par deux caractéristiques. D'un côté, le programme prévoit une enveloppe de 700 millions d'euros permettant de soutenir financièrement la mise en place de projets d'ÉcoCités. De l'autre, ce programme met fin à la mainmise du ministère sur les politiques urbaines d'environnement. Celui-ci doit désormais compter sur la CDC et surtout sur l'influent Commissariat général à l'investissement (CGI), rattaché directement au Premier ministre, qui s'avère être le véritable maître du jeu du Programme Ville de demain, notamment en ce qui concerne la rédaction du cahier des charges sur la base duquel sont sélectionnés et évalués les projets locaux. Le CGl est également le principal promoteur d'une approche laissant une large place aux recettes néomanagériales pour contraindre les villes à s'adapter aux cadres imposés par l'État. C'est ce que souligne Raphaël Frétigny :

«L'appel à projet met en concurrence les projets d'ÉcoCités pour l'allocation des crédits du Grand Emprunt. Les actions qui seront sélectionnées seront celles qui correspondent le mieux aux attentes du cahier des charges national, définies à Paris par le CGI, en collaboration avec le MEEDDM et la CDC. Cette mise en concurrence est affirmée fortement par le CGl, qui rappelle que "les financements iront aux opérations les plus performantes. II n'y aura pas de montant fixe pour chaque ville, et il pourra y avoir une différence forte dans les montants entre les villes". Dès lors, les villes ont intérêt à présenter des dossiers de candidature qui correspondent le mieux possible aux critères fixés dans le cahier des charges, sous peine de se voir exclues de la distribution des crédits » (Frétigny, 2010, p. 113).

Ce caractère plus coercitif du programme Ville de demain se retrouve dans le cahier des charges élaboré par le CGI qui soumet les candidats à un impératif de performance :

"Les actions susceptibles d'être financées par le fonds "Ville de demain" devront répondre à un haut niveau de performance environnementale et d'innovation et avoir un fort caractère démonstrateur. II s'agit là de prouver, par des réalisations concrètes, la faisabilité des opérations et d'en tirer un maximum d'enseignements en vue d'une diffusion plus large. Le fonds "Ville de demain" s'inscrit ainsi dans un processus fortement sélectif » (CGl, 2010, p. 9).

Cette sélectivité et ce souci de la performance a donné lieu à des réflexions concernant l'établissement d'un lien entre performance et budgétisation, qui se matérialise notamment

15. En 2011, six nouvelles ÉcoCités sont invitées à intégrer la démarche, portant le nombre total de projets retenus à 19 (18 pour la France métropolitaine, dont 3 pour le Grand Paris, et 1 pour la Réunion). Ces projets se démarquent des écoquartiers dans la mesure où ils portent sur des espaces plus importants, allant du grand quartier à l'aire urbaine. 
dans le contrôle strict des 14 indicateurs de sélection et de suivi des projets. Ainsi, le programme Ville de demain permet à l'État d'orienter les acteurs locaux vers ses priorités en finançant sélectivement certaines opérations d'ingénierie (îlots urbains intégrés, smart grids, constructions exemplaires, etc.). Seuls les projets se conformant au cahier des charges du CGI sont financés, permettant ainsi d'opérer un tri entre les projets développement durable favorisant les actions misant sur la performance et les innovations technologiques et les autres. Dans le même temps, le ministère et surtout les services de la CDC - le Département politique de la ville et développement urbaine (DPVDU) et la direction du Développement territorial et du Réseau (DDTR) - sont en charge de la mise en réseau des acteurs et de la capitalisation des expériences. Après avoir mis à disposition des crédits d'ingénierie pour le financement d'études ou d'assistance à maîtrise d'ouvrage, ces acteurs ont identifié pour chaque thématique des expériences pilotes. Ainsi, Strasbourg, Montpellier et Bordeaux sont mis en avant pour les îlots urbains intégrés, Plaine Commune, Nantes et Marseille pour la gestion des ressources et le recyclage des matériaux, Grenoble et Issy-les-Moulineaux pour les smart grids. Ces projets font alors l'objet d'un travail visant à dégager leurs caractéristiques principales en vue de faciliter leur reproduction dans d'autres territoires.

Trois conclusions peuvent être tirées de cette analyse du plan Ville durable. Premièrement, même en l'absence d'incitation financière, les villes se sont mobilisées pour répondre aux appels à projet. De l'avis des acteurs locaux, il s'agit de « prendre le bon wagon » pour valoriser leurs démarches, leur donner de la visibilité et surtout bénéficier d'une implication dans les réseaux du ministère qui pourrait à terme être pourvoyeuse de bénéfices (accéder à des informations sur un domaine dans lequel la réglementation est en évolution rapide, capter des financements futurs, influencer les cadres d'action nationaux). La mise en place du Programme ville de demain semble avoir donné raison a posteriori à ces stratégies de positionnement, qui occupent aujourd'hui une place importante dans certaines villes comme Lyon ou Nantes (Béal, 2011). Deuxièmement, ces programmes signent un retour paradoxal de l'État. Comme l'ont souligné d'autres travaux, la réforme néomanagériale de l'État dans les domaines de l'environnement et du développement durable reste ambiguë (Lascoumes et al., 2014). L'État a certes repris la main sur les politiques urbaines de développement durable en parvenant à orienter les projets urbains vers ses priorités (limitation des émissions de gaz à effet de serre, écoconstruction, et plus généralement développement d'une écologie high-tech) et à construire des référentiels et labels. Toutefois, cette reprise en main s'appuie sur les démarches des villes (ou plutôt de certaines d'entre elles) qui ont été identifiées comme les plus innovantes. On assiste donc à une co-construction locale/nationale des contenus des politiques urbaines dans laquelle l'État joue un rôle d'« uploader» qui sélectionne, capitalise et diffuse des pratiques. Enfin, troisièmement, ces programmes n'ont pas uniquement une visée domestique, dans la mesure où ils prennent également sens à l'échelle internationale. Dans un contexte où l'environnement et la durabilité urbaine constituent l'un des marchés les plus porteurs, notamment dans les pays des Suds (Hult, 2015), l'État cherche à "vendre " ses bonnes pratiques à l'international. L'action de l'ADEME dans les pays du Maghreb (Barthel, 2014), celle du ministère en Chine ou encore la création de la marque Vivapolis ${ }^{16}$ sont des signes de cette montée

16. La marque Vivapolis et son slogan « Powered by French Creativity » ont été lancés en 2013 par Nicole Bricq, alors ministre du Commerce extérieur. Portée par le Comité stratégique de filière éco-industrielle (COSEI), elle cherche à promouvoir les champions français de la ville durable à l'étranger. 
en puissance de l'État dans les circulations et les transferts nationaux et internationaux, montée en puissance qui sert des objectifs politiques de reconstruction d'une capacité à intervenir dans les territoires et également des objectifs économiques d'exportation des projets " vitrines " et des entreprises françaises à l'international.

Avant de revenir dans la conclusion générale sur ce que ces deux cas nous disent de la contribution des circulations à la transformation des rapports entre l'État et les villes, attardonsnous sur un certain nombre de similitudes et de différences. Dans les deux cas, l'intensification des circulations s'inscrit dans un processus de réorganisation néomanagériale de l'action de l'État qui fait la part belle à des instruments tels que les appels à projets ou la distribution de labels. Avec ces nouveaux instruments, l'État reconnaît l'autonomie des villes, leur expertise et leur capacité d'innovation, et renforce la visibilité nationale et internationale de certaines d'entre elles. Mais ces instruments permettent surtout à l'État de récupérer une prise sur les contenus des politiques publiques en mettant les villes en concurrence et en sélectionnant et certifiant certains projets jugés innovants et se conformant à ses priorités d'action. Toutefois, des différences assez nettes apparaissent également entre les deux programmes. L'influence de cet État régulateur est plus nette dans les politiques de rénovation urbaine que dans les politiques de développement durable. La raison est sans doute à trouver dans le caractère plus ancien de la politique de la Ville qui a permis aux agents de l'État de construire et de raffiner une doctrine d'action qui semble encore balbutiante dans le cas du développement urbain durable. Ensuite, le caractère coercitif de la diffusion des modèles semble plus net dans le PNRU qui promeut une action davantage standardisée que celle proposée par le plan Ville durable. Dans le même sens, la sélection et la diffusion de modèles par l'État dans le domaine de la rénovation urbaine prend uniquement sens dans la sphère domestique, tandis que celle qui est organisée autour du développement durable dispose également d'une dimension internationale forte.

\section{Conclusion}

L'analyse des circulations de modèles et des «bonnes pratiques » en matière de politique urbaine montre clairement que les deux thèses de la montée en puissance des villes comme acteurs et espaces politiques et du repositionnement de l'État dans la fabrique des politiques territoriales, longtemps tenues pour antagonistes, sont en réalité parfaitement compatibles et correspondent à deux réalités empiriques articulées l'une avec l'autre. Une des raisons de l'antagonisme dans lequel ces deux thèses ont longtemps été tenues tient sans doute au décalage temporel qui a vu, d'abord, les grandes villes s'engouffrer dans la brèche ouverte par la décentralisation, la construction européenne et la mondialisation qui ont toutes trois déstabilisé l'administration d'État et son rapport au(x) territoire(s) et, ensuite, l'État réinventer ses modes d'intervention et d'influence. Aujourd'hui, un nouveau modèle de relation entre État et villes semble ainsi s'être (provisoirement ?) stabilisé en France qui voit ces dernières être de plus en plus chargées de définir les champs, priorités et outils d'action publique urbaine et le premier faire le tri entre ces innovations locales et transformer les plus prometteuses d'entre elles en normes diffusées ensuite au travers des appels à projet compétitifs et des labels.

II n'existe pas encore, à notre connaissance du moins, de travaux qui nous permettraient de savoir si cette mutation de l'État en acteur qui régule des secteurs d'action publique en 
érigeant des initiatives locales en références (qui passe du référentiel aux « références » en somme) est le produit d'une stratégie concertée et délibérée ou le résultat de tâtonnements contingents. En attendant, on peut constater que la France n'est pas le seul pays concerné par cette évolution. D'une certaine manière, l'Union européenne a procédé de la même manière dans des secteurs d'action publique comme l'énergie ou le genre où sa légitimité et sa compétence n'étaient pas pleinement reconnues (Sabel, Zeitlin, 2012). Sous les gouvernements Blair et Brown, le Royaume-Uni a aussi donné des signes d'évolution similaires. Par exemple, dans le cadre du programme Millenium Community lancé en 1999, l'État britannique a cherché à susciter des innovations environnementales dans les politiques urbaines en identifiant et consacrant sept projets jugés «exemplaires » dans des domaines précis (normes dans la construction, performance énergétique, mobilités douces, espaces publics, etc.) et susceptibles de servir de modèle pour les autres villes.

Ce modèle de relations entre l'État et les villes est-il appelé à se généraliser? Rien n'est moins sûr! Tous les programmes territoriaux de l'État n'ont pas basculé dans un régime de "gouvernement à distance » forgé dans un domaine de l'action publique - la Politique de la Ville - à forte portée symbolique et politique (Epstein, 2013a). II en va probablement de même du gouvernement par les modèles ici esquissés, qui ne concernent pour l'heure que des secteurs et enjeux «nouveaux », peu stabilisés tels que la construction durable ou l'économie numérique. Ces «domaines » sont encore peu saturés en acteurs, intérêts constitués ou expertise établie. L'État et ses grands corps ne peuvent guère y faire prévaloir de visions, de savoirs et d'intérêts précis. En même temps, l'État peut difficilement ne pas avoir de discours volontariste sur ces secteurs. La sélection et la promotion des modèles locaux a donc le double avantage de créditer l'État d'un volontarisme sur des thèmes innovants tout en fournissant à peu de frais les linéaments d'un référentiel. Dans un contexte de crise des finances publiques, l'intérêt pour l'État saute aux yeux. Ces considérations ne sont sans doute pas étrangères à la récente création d'un Institut de la ville durable ${ }^{17}$ dont la préfiguration a été confiée à l'ANRU et qui " doit permettre que l'État reste garant de la politique publique menée » en matière d'urbanisme durable et de rénovation urbaine. Comme dans d'autres domaines, l'État délègue non plus seulement la mise en œuvre des politiques mais aussi l'innovation en la matière, tout en cherchant à rester maître de sa mise en forme et bénéficiaire des bénéfices politiques qui lui sont associés.

Vincent Béal

Université de Strasbourg

SAGE (UMR 7363)

Faculté des sciences sociales vbeal@unistra.fr

Renaud Epstein

Université de Nantes

Faculté de droit et des sciences politiques renaud.epstein@univ-nantes.fr

\author{
Gilles Pinson \\ Sciences Po Bordeaux \\ Centre Émile Durkheim \\ g.pinson@sciencespobordeaux.fr
}

17. [http//www.ville.gouv.fr/IMG/pdf/rapportpeylet.pdf]. 


\section{Bibliographie}

Ancelovici, M., Jenson, J. (2012), « La standardisation et les mécanismes du transfert transnational ", Gouvernement et action publique, 1 (1), p. 37-58.

BARTHEL, P.-A. (2014), « L'exportation au Maroc de la "ville durable" à la française ", Métropolitiques, 16 juin [http://www.metropolitiques.eu/L-exportation-auMaroc-de-la-ville.html].

Beal, V. (2011), Les Politiques du développement durable. Gouverner l'environnement dans les villes françaises et britanniques (1970-2010), thèse de doctorat en science politique. Université de Lyon-Saint-Étienne.

BÉAL, V. (2014), " "Trendsetting cities" : les modèles à l'heure des politiques urbaines néo-libérales ", Métropolitiques, 30 juin [http://www.metropolitiques.eu/ Trendsetting-cities-les-modeles-a.html].

BeAL, V., PInson, G. (2015), "From the Governance of Sustainability to the Management of Climate Change: Reshaping Urban Policies and Central-local Relationships in France ", Journal of Environmental Policy and Planning, 17 (3), p. 402-419.

Benington, J., Harvey, J. (1999), "Networking in Europe ", in STOKER, G. (ed.), The New Management of British Local Governance, Basingstoke, PalgraveMacmillan, p. 197-221.

Benson, D., Jordan, A. (2012), "Policy Transfer Research: Still Evolving, not yet Through? ", Political Studies Review, 10 (3), p. 333-338.

Bergeron, H., Castel, P., Dubuisson-Quellier, S. (2014), « Gouverner par les labels. Une comparaison des politiques de l'obésité et de la consommation durable ", Gouvernement et action publique, 3 (3), p. 7-31.

Bezes, P., Le LIDEC, P. (2011a), "Steering from the Centre in France in the 2000s: When Reorganizations Meet Politicization ", in Dahlström, C., Peters, G., Pierre, J. (eds), Steering from the Centre, Toronto, University of Toronto Press, p. 27-53.

Bezes, P., Le LIDEC, P. (2011b), «L'hybridation du modèle territorial français ", Revue française d'administration publique, 136 (4), p. 919-942.

BILlAND, E. (2012), La Fonction publique territoriale, Paris, La Découverte.

Borraz, O. (1998), Gouverner une ville: Besançon, 1959-1989, Rennes, Presses universitaires de Rennes.

Brenner, N. (2004), New State Spaces: Urban Restructuring and State Rescaling in Western Europe, Oxford, Oxford University Press.
BREton, E. (2014), « Répondre à l'appel (à projets). Récits d'un apprentissage silencieux des normes de l'action publique patrimoniale ", Politix, 105, p. 213-232.

Bruno, I., DidieR, E. (2013), Benchmarking : l'État sous pression statistique, Paris, Zones.

Carriou, C., Ratouis, O. (2014), "Actualité des modèles urbanistiques ", Métropolitiques, 18 juin [http://www.metropolitiques.eu/Actualite-desmodeles.html].

CGI (2010), Investissement d'avenir : Ville de demain, Cahier des charges, volet 1.

Claude, V., Saunier, P. Y. (1999), "L'urbanisme au début du siècle. De la réforme urbaine à la compétence technique ", Vingtième siecle. Revue d'histoire, 64 (4), p. 25-39.

Crozier, M., Thoenig, J.-C. (1975), "La régulation des systèmes organisés complexes : le cas du système de décision politico-administratif local en France", Revue française de sociologie, 16 (1), p. 3-32.

DALN (2011), Pour une ville durable, direction générale de l'Aménagement, du Logement et de la Nature.

Debar, A. (2013), "Les directeurs départementaux sont-ils encore les "patrons" de l'État local ? ", Sciences de la société, 90, p. 42-57.

DeLPEUCH, T. (2008), « L'analyse des transferts internationaux de politiques publiques : un état de l'art ", Questions de recherche, 27, p. 1-69.

Devisme, L., Dumont, M., Roy, E. (2007), « Le jeu des "bonnes pratiques" dans les opérations urbaines, entre normes et fabrique locale", Espaces et sociétés, 131 (4), p. 15-31.

DolowitZ, D., MARSH, D. (2000), "Learning from Abroad: The Role of Policy Transfer in Contemporary Policy-Making », Governance, 13 (1), p. 5-24.

Donzelot, J., Estebe, P. (1994), L'État animateur. Essai sur la politique de la ville, Paris, Seuil.

DuFlot, C. (2012), Discours de lancement du Label national ÉcoQuartier, Brétigny-sur-Orge, 12 décembre 2014.

Dupuy, C., Pollard, J. (2014), «A Dethroned King? The Limits of State Infrastructural Power in France, Public Administration, 92 (2), p. 359-374.

Epstein, R. (2008), Gouverner à distance. La rénovation urbaine, démolition-reconstruction de l'appareil d'État, thèse de sociologie de l'ENS Cachan. 
EPSTeIN, R. (2013a), La Rénovation urbaine : démolition-reconstruction de l'État, Paris, Presses de Sciences Po.

EPSTEIN, R. (2013b), « L'État local, de la résistance à la résidualisation. Les services extérieurs à l'épreuve des réformes administratives ", in EYMERI-DOUZANS, J.-M., BouckaerT, G. (dir.), La France et ses Administrations : un état des savoirs, Bruxelles, Bruylant, p. 585-603.

Evans, M. (2004), Policy Transfer in Global Perspective, Aldershot, Ashgate.

Foucault, M. (1975), Surveiller et punir. Naissance de la prison, Paris, Gallimard.

Frébault, J., Lemosquet, J.-C. (dir.) (1993), Le Renouveau de la planification urbaine et territoriale, Paris-la-Défense, MELT, DAU.

Fretigny, R. (2010), Le Développement urbain durable dans les grandes agglomérations françaises: projets locaux et interventions d'État. De l'accompagnement des projets à leur mise en programme, mémoire de master 2 en sociologie, ENS Cachan.

Gaudin, J.-P. (1985), L'Avenir en plan : technique et politique dans la prévision urbaine, 1900-1930, Seyssel, Éditions Champ Vallon.

Gremion, P. (1976), Le Pouvoir périphérique: bureaucrates et notable dans le système politique français, Paris, Seuil.

HASSENTEufel, P. (2005), « De la comparaison internationale à la comparaison transnationale ", Revue française de science politique, 55 (1), p. 113-132.

HAssenteufel, P. (2008), "L'État mis à nu par les politiques publiques?", dans BADIE, B., Deloye, Y. (dir.), Les Temps de l'État, Paris, Fayard, p. 311-329.

Hult, A. (2015), "The Circulation of Swedish Urban Sustainability Practices: to China and Back ", Environment and Planning A, 47 (3), p. 537-553.

IHL, O. (2007), Le Mérite et la République. Essai sur la société des émules, Paris, Gallimard.

Kaluszynski, M., PAYre, R. (dir.) (2013), Savoirs de gouvernement, Paris, Economica.

Lascoumes, P., Le Galès, P. (dir.) (2004), Gouverner par les instruments, Paris, Presses de Sciences Po.

Lascoumes, P., Bonnaud, L., Le Bourhis, J.-P., MARTINAIS, E. (2014), Le Développement durable: une nouvelle affaire d'État, Paris, Presses universitaires de France.

LE GaLÈs, P. (1999), " Le desserrement du verrou de l'État?", Revue internationale de politique comparée, 6, p. 627-652.

Le GalÈs, P. (2011), Le Retour des villes européennes. Sociétés urbaines, mondialisation, gouvernement et gouvernance, Paris, Presses de Sciences Po [2 $2^{\ominus}$ éd.].

LE GalÈs, P. (2013), "La gouvernance territoriale après la crise", dans PASQUieR, R., SIMOULIN, V., WEISBEIN, J. (dir.), La Gouvernance territoriale : pratiques, discours et théories, Paris, LGDJ, p. 289-300.

LE LIDEC, P. (2007), « Le jeu du compromis : l'État et les collectivités territoriales dans la décentralisation en France", Revue française d'administration publique, 121-122, p. 111-130.

LORRAIN, D. (1991), " De l'administration républicaine au gouvernement urbain ", Sociologie du travail, 33 (4), p. 461-484.

MAILLARD, J. DE (2004), Réformer l'action publique: la politique de la ville et les banlieues, Paris, LGDJ.

McCANn, E., WARD, K. (eds) (2011), Mobile Urbanism: City Policymaking in the Global Age, Minneapolis (Minn.), University of Minnesota Press.

McCANn, E., WARD, K. (2012), « Policy Assemblages, Mobilities and Mutations: Toward a Multidisciplinary Conversation ", Political Studies Review, 10 (3), p. 325-332.

McFarlane, C. (2011), "Assemblage and Critical Urbanism », City, 15, p. 204-224.

Merlin, P. (2010), Les Grands Ensembles. Des discours utopiques aux " quartiers sensibles ", Paris, La Documentation française.

METL (2012), Dossier de presse du lancement du label national ÉcoQuartier, Paris, ministère de l'Égalité des Territoires et du Logement.

Moran, M. (2002), "Understanding the Regulatory State ", British Journal of Political Science, 32 (2), p. 391-413.

Oliver, J., LODGE, M. (2003), "The Limitations of "Policy Transfer" and "Lesson Drawing" for Public Policy Research ", Political Studies Review, 1 (2), p. 179-193.

OFFNER, J.-M. (1988), "L'expertise locale en transports urbains: entre logiques professionnelles et organisationnelles ", Politiques et management public, 6 (1), p. 81-102.

Pasquier, R., Pinson, G. (2004), "Politique européenne de la ville et gouvernement local en Espagne et en Italie ", Politique européenne, 1, p. 42-65.

PAyre, R. (2010), "The Importance of Being Connected. City Networks and Urban Government: Lyon and Eurocities (1990-2005) ", International Journal of Urban and Regional Research, 34 (2), p. 260-280.

Peck, J., Theodore, N. (2015), Fast Policy: Experimentalist Statecraft at the Thresholds of Neoliberalism, Minneapolis (Minn.), University of Minnesota Press. 
PeCK, J. (2011), "Geographies of Policy: From Transfer-Diffusion to Mobility-Mutation ", Progress in Human Geography, 35 (6), p. 773-797.

Peyroux, E., Putz, R., Glasze, G. (2012), « Business Improvement District (BIDs), The Internationalization and Contextualization of a "Travelling Concept" ", European Urban and Regional Studies, 19 (2), p. 111-120.

PINson, G. (2009), Gouverner la ville par projet : urbanisme et gouvernance des villes européennes, Paris, Presses de Sciences Po.

Poupeau, F.-M. (2013), "L'émergence d'un État régional pilote. La recomposition des jeux administratifs autour du ministère de l'Écologie et du Développement durable dans une région française ", Gouvernement et action publique, 2 (2), p. 249-277.

RadAeLLI, C. (2000), « Policy Transfer in the European Union: Institutional Isomorphism as a Source of Legitimacy ", Governance, 13 (1), p. 25-43.

Robinson, J. (2011), "The Spaces of Circulating Knowledge: City Strategies and Global Urban Governmentality ", in McCANn, E., WARD, K. (eds),
Mobile Urbanism: Cities and Policy-making in the Global Age, Minneapolis (Minn.), University of Minnesota Press, p. 15-39.

SABel, C. F., ZeitLin, J. (eds) (2012), Experimentalist Governance in the European Union, Oxford, Oxford University Press.

SAUNIER, P. Y. (2006), " La toile municipale auX XIX $x^{-}-X X^{\circ}$ siècles: un panorama transnational vu d'Europe ", Urban History Review/Revue d'histoire urbaine, 34 (2), p. 43-56.

Soderstrom, O., Paquot, T. (2012), "Modèles urbains ", Urbanisme, 383, p. 41-42.

THoEnIG, J.-C. (1973), L'Ére des technocrates, Paris, Les Éditions d'organisation.

VION, A. (2003), "L'invention de la tradition des jumelages (1951-1956): mobilisations pour un droit ", Revue française de science politique, $53(4)$, p. 559-582.

Wolman, H., Page, E. (2002), "Policy Transfer among Local Governments: An Information-Theory Approach ", Governance, 15 (4), p. 477-501. 